\section{Michelle Debatisse Franck Tolédo}

\title{
Amplification génique, plasticité des génomes et oncogenèse
}

L'accumulation de copies surnuméraires d'un segment de chromosome engendre instabilité et réarrangements génomiques précurseurs de l'oncogenèse. L'étude des premiers stades de l'amplification génique chez les mammifères, grâce à la technique d'hybridation de sondes fluorescentes in situ (FISH), a montré qu'une large part de l'instabilité génomique est corrélée à des processus intrachromosomiques, comportant la cassure d'une chromatide puis la fusion, après réplication, entre les chromatides sœurs. Ce phénomène devient cyclique car le pont qui se forme entre les deux chromatides engendre des cassures lors des mitoses. L'amplification génique extrachromosomique pourrait procéder de l'excision d'une grande molécule circulaire acentrique dont les copies seraient ensuite amplifiées, au cours des divisions mitotiques, par ségrégation inégale entre les cellules sœurs. Des délétions, parallèles à l'amplification, pourraient être responsables de la perte de gènes suppresseurs de tumeurs. L'ensemble de ces phénomènes concourt à l'installation des processus malins.



amplification génique est un phénomène qui conduit une cellule à accumuler des copies multiples d'une fraction limitée de son génome. Si la cellule concernée par cet événement mutationnel en retire un avantage sélectif, sa descendance pourra envahir la population cellulaire. Cette succession d'événements, bien caractérisés chez les mammifères avec des systèmes modèles de cellules cultivées in vitro, s'avère d'une importance clinique considérable. En effet, il est clair actuellement que l'amplification de divers oncogènes est corrélée à la progression d'un grand nombre de tumeurs [1]. Récemment, il a été montré que les cellules ne sont pas toutes capables d'amplification et un certain nombre des facteurs qui déterminent cette aptitude ont été caractérisés. Les mécanismes moléculaires mis en jeu pour accumuler les copies surnuméraires ont également été partiellement élucidés. De nombreuses études cytogénétiques ont montré que, dans les populations de cellules néoplasiques et prénéoplasiques, au contraire des cellules normales, des réarrangements génomiques variés tels que délétions, translocations, chromosomes dicentriques et aneuploïdies, sont fréquents. Des résultats récents suggèrent que les événements d'amplification et ces autres réarrangements obéissent à un déterminisme génétique commun. 


\section{Amplifier ou ne pas amplifier?}

L'amplification d'oncogènes n'est jamais trouvée dans les tissus normaux des malades alors qu'elle a souvent été détectée au niveau des cellules tumorales (pour revue, voir [2]). In vitro, des mutants résistants à diverses substances cytotoxiques, par amplification du gène qui code pour la protéine cible de ces inhibiteurs, sont obtenus à relativement grande fréquence à partir de lignées de cellules tumorales, transformées ou immortelles, mais pas à partir de cellules normales [3,4]. La similitude des résultats obtenus in vivo et in vitro permet, en outre, de penser que les systèmes de cellules en culture constituent des modèles adéquats pour l'analyse des facteurs qui contrôlent la régulation de l'amplification. Récemment, certains intermédiaires moléculaires impliqués dans la permissivité pour l'amplification ont été caractérisés, et il a été établi qu'ils participent également à la régulation de la progression dans le cycle cellulaire. C'est notamment le cas de la protéine p53, codée par le gène suppresseur de tumeur le plus fréquemment inactivé dans les tumeurs humaines (pour revue voir [5]). Les cellules portant une mutation récessive sur chaque allèle du gène qui code pour p53 sont permissives pour l'amplification, et la réintroduction par transfection d'un allèle normal suffit à supprimer cette propriété [6, 7]. Par ailleurs, lorsque la protéine p53 s'accumule dans des cellules normales, par exemple en réponse à la présence de cassures dans leur ADN, l'expression de divers gènes est induite. L'un d'eux code pour une protéine régulatrice nommée p21, Cipl ou WAF1, un inhibiteur de kinases dépendantes de cyclines (Cdk) $\left(m / s n^{\circ} 2\right.$, vol. 10, p. 206; $n^{\circ} 6-7$ vol. 10, p. 744) (pour revues, voir $[8$, 9]) qui bloque la progression dans le cycle au point de contrôle Gl/S [10]. Le fait que la protéine p21 se comporte comme un suppresseur du phénotype tumoral in vitro [11] permet d'établir une relation directe entre contrôle du cycle cellulaire, oncogenèse et capacité d'amplifier le génome. La répression de l'amplification n'est probablement pas effectuée par les seuls intermédiaires de la voie de régulation à laquelle participe p53, l'analyse des possibilités alternatives de contrôle est en cours dans plusieurs laboratoires, mais encore un peu préliminaire pour être détaillée ici.

\section{Comment amplifier? Un peu d'histoire}

La reconstitution des mécanismes d'amplification a été abordée depuis longtemps en analysant la localisation des copies surnuméraires des gènes dans des cellules de lignées fortement amplifiées. L'étude cytologique a montré (pour revue, voir [12]): (1) que les séquences amplifiées sont de grande taille, variant de quelques centaines à quelques milliers de kilobases; (2) qu'elles peuvent être localisées, soit au niveau d'éléments extra-chromosomiques circulaires et dépourvus de centromère, appelés "double minutes" (DM), soit au niveau d'expansions chromosomiques, initialement caractérisées par un spectre de bande particulier (HSR : homogeneously staining regions); (3) que les HSR sont trouvées souvent, bien que non systématiquement, sur un bras de chromosome qui porte le locus considéré dans les cellules non amplifiées. Ces observations ont été à la base d'une controverse qui a duré une dizaine d'années, visant à définir la séquence des événements susceptibles de conduire à ces deux situations: certains auteurs pensaient que l'amplification survenait d'abord in loco et qu'une pulvérisation secondaire des HSR pouvait engendrer les DM, d'autres suggéraient que les DM représentaient, au contraire, les structures initiales, et que leur réintégration éventuelle dans les chromosomes pouvait donner naissance aux HSR (pour revue, voir [13]). Cette dernière hypothèse était étayée par un certain nombre d'études montrant que la propagation in vitro de cellules tumorales porteuses de DM pouvait aboutir à un envahissement progressif de la population par des cellules possédant une HSR en position ectopique. Cependant, l'ensemble des résultats obtenus en analysant différents systèmes ne s'explique pas aisément par cette seule succession d'événements. L'analyse par de nombreux groupes de l'organisation des répétitions au sein des structures amplifiées (pour
GM. Re mechanisms of mammalian DNA amplification. Cell $1989 ; 57: 901-8$. 



Figure 1. Modèle des "peaux d'oignon ". Des redémarrages multiples au niveau d'une origine de réplication créent une structure localement polytène. En noir sont représentés les produits normaux de la réplication, en rouge les séquences amplifiées résultant de la surréplication. La structure devra ensuite être remodelée pour traverser la mitose.

revue, voir [13]) a fourni des résultats complexes, établissant que chaque mutant a des caractéristiques qui lui sont propres. Cette situation peut s'interpréter de deux façons: soit des mécanismes d'amplification variés opèrent dans les cellules de mammifères, soit le mécanisme mis en jeu pour l'acquisition des copies excédentaires est en lui-même source d'instabilité, ces deux possibilités ne s'excluant d'ailleurs pas. La première de ces hypothèses a reçu un soutien expérimental par la démonstration que des mécanismes d'amplification différents fonctionnent dans des systèmes biologiques particuliers. Quel- ques cas d'amplification participant à un programme de développement ont été mis en évidence chez certains eucaryotes, par exemple l'amplification des gènes qui codent pour les ARN ribosomiques dans les ovocytes de xénope et pour les protéines du chorion dans les cellules folliculaires de drosophile. Dans ces systèmes, toutes les cellules qui ont atteint le stade de différenciation concerné subissent l'événement de façon synchrone, et les structures formées sont immédiatement accessibles à l'observation. L'analyse par microscopie électronique, entre autres techniques utilisées, a permis de montrer que les copies surnuméraires proviennent d'une surréplication locale qui résulte respectivement de la mise en œuvre de cercles roulants extrachromosomiques ou de multiples réamorçages de la réplication sans division cellulaire (pour revue, voir [14]). Ce dernier mécanisme était considéré encore récemment comme le plus probable dans les cellules de mammifères, bien que la structure localement polytène créée par ces réamorçages soit instable au cours de la mitose. Cela ne pose aucun problème au niveau des cellules folliculaires qui ne se divisent plus, mais des événements de recombinaison multiples seraient nécessaires pour que les copies surnuméraires deviennent transmissibles aux cellules filles dans le cas de cellules en croissance (figure 1). L'aspect le plus attrayant de ce modèle dit des "peaux d'oignon", était que le produit du mécanisme d'amplification constitue, par luimême, une source d'instabilité et peut donc rendre compte de la grande variété des situations observées dans les cellules de mammifères [15]. Cependant, aucune des hypothèses concernant les modalités de résolution de cette structure complexe n'a pu être testée expérimentalement.

Le concept d'instabilité des structures initiales a conduit plusieurs groupes à reconsidérer l'approche expérimentale utilisée jusque-là pour analyser ce phénomène. En effet, si les produits initiaux de l'amplification sont fréquemment remodelés et si un avantage de croissance est acquis de ce fait par certaines cellules, les lignées mutantes seront généralement constituées de cellules où les structures engendrées par des réarrangements secondaires masqueront les produits directs du mécanisme d'amplification. Il était donc crucial d'étudier l'organisation des copies surnuméraires dans des mutants apparus récemment [13]. Un protocole satisfaisant à cette exigence a été élaboré : il consiste, avant de semer les cellules en milieu sélectif, à isoler de petites sous-populations statistiquement dépourvues de mutants préexistants et à les cultiver en milieu non sélectif pendant un nombre limité de générations pour permettre l'apparition de nouveaux mutants [16]. Le développement de la technique d'hybridation in situ avec des sondes froides révélées par des anticorps fluorescents (FISH), suffisamment puissante pour être appliquée à un très petit nombre de cellules peu amplifiées, a ensuite permis une analyse précoce des colonies résistantes. Des cellules de hamster ayant une amplification des gènes codant pour la dihydrof olate réductase (DHFR) [17-19], l'aspartate transcarbamylase (CAD) [20,21], et l'adénylate désaminase (AMPD) [22-24] ont ainsi été analysées, généralement dix à vingt générations seulement après l'événement initial. Les résultats obtenus dans ces conditions ont profondément bouleversé les idées en cours et il semble s'établir maintenant un consensus pour considérer que, dans les cellules de mammifères, l'amplification résulte d'une ségrégation inégale des copies lors des mitoses successives, plutôt que d'une surréplication locale. En outre, deux mécanismes indépendants au moins peuvent fonctionner dans les cellules d'une même lignée. Ils accumulent des copies du gène sélectionné, respectivement sur le bras chromosomique d'origine ou sur des DM; ces conclusions restent compatibles avec l'observation que des réintégrations secondaires des DM peuvent survenir dans certains cas.

\section{Un mécanisme initial intrachromosomique}

L'amplification in trachromosomique a été étudiée pour des mutants récents dans les trois systèmes cités au paragraphe précédent. Les premières analyses ont montré que l'un des deux bras chromosomiques por- 


\section{RÉFÉRENCES}

14. Kafatos FC, Orr W, Delidakis C. Developmentally regulated gene amplification. Trends Genet 1985; 1 : 301-6.

15. Schimke RT, Sherwood SW, Hill AB, Johnston RN. Overreplication and recombination of DNA in higher eukaryotes: potential consequences and biological implications. Proc Nall Acad Sci USA 1986; 83: $2157-61$.

16. Giulotto E, Saito I, Stark (:R. Structure of DNA formed in the first step of CAD gene amplification. EMBO J 1986; 5 : 2115 gien.

17. Trask BJ, Hamlin JL. Early dihydrofolate reductase gene amplification events in $\mathrm{CHO}$ cells usually occur on the same chromosome arm as the original locus. Genes Dev $1989 ; 3: 1913-25$.

18. Windle B, Draper BW, Yin Y, O'Gorman $S$, Wahl GM. A central role for chromosome breakage in gene amplification, deletion formation and amplicon integration. Genes Dev $1991 ; 5: 160-74$

19. Ma C, Martin S, Trask B, Hamlin JL. Sister chromatid fusion initiates amplification of the dihydrofolate reductase gene in Chinese hamster cells. Genes Dev 1993; 7: 60520.

20. Smith KA, Gorman PA, Stark MB, Groves RP, Stark GR. Distinctive chromosomal structures are formed very early in the amplification of CAD genes in Syrian ham ster cells. Cell 1990; 63: 1219-27.

21. Smith KA, Stark MB, Gorman PA, Stark GR. Fusion near telomeres occurs very early in the amplification of CAD genes in Syrian hamster cells. Proc Natl Acad Sri USA 1992 89: 5427-31.

22. Toledo F, Smith KA, Buttin G, Debatisse M. The evolution of the amplified adenyla te-deaminase-2 domains in Chinese hamste cells suggests the sequential intervention of different mechanisms of DNA amplification. Mutat Res 1992; 276: 261-73.

23. Toledo F, LeRoscouet D, Buttin G, Debatisse M. Co-amplified markers alternate in megabase long chromosomal inverted repeats and cluster independently in interphase nuclei at early steps of mammalian gene amplification. EMBO J 1992; 11 : 266573.

24. Toledo F, Buttin G, Debatisse M. The origin of chromosome rearrangements at early stages of $A M P D 2$ gene amplification in Chinese hamster cells. Curr Biol 1993; 3: tant le gène dans les cellules non amplifiées est normal, l'autre porte les copies excédentaires; ce qui suggère un processus initial concernant un seul homologue $[17,20,22]$. Bien que des populations clonales aient été généralement étudiées, une distribution remarquablement hétérogène des unités amplifiées en nombre et en taille a été observée d'une cellule à l'autre dans des mutants analysés une vingtaine de générations après l'événement initial $[20,22]$. Deux types majeurs de structures ont été trouvés répétitivement sur l'homologue amplifié: une structure en échelle comprenant de deux à une dizaine de copies du gène sélectionné, espacées généralement de plusieurs mégabases et une structure compacte composée d'un plus grand nombre de répétitions de taille réduite. Les structures en échelle n'ont été observées qu'aux stades les plus précoces, alors que la structure compacte se substitue progressivement aux autres au cours de l'expansion clonale. L'observation de mutants à des stades plus tardifs permet de penser que, dans chaque clone, une sous-population de cellules portant une structure condensée et stabilisée envahit la population, aboutissant à une organisation et un nombre de copies homogènes de cellule à cellule $[20,22]$. Pour déterminer si les unités géantes précoces sont associées en orientation directe ou inverse, des expériences de FISH avec des combinaisons de sondes révélées par des fluorochromes différents ont été effectuées. Pour les systèmes DHFR et AMPD, des sondes ont été obtenues pour des loci potentiellement coamplifiables avec le gène sélectionné, mais séparés de celui-ci par une dizaine de $\mathrm{Mb}$ afin que les deux séquences soient individualisées cytologiquement sur les chromosomes métaphasiques. Il a d'abord été montré dans le cas de l'amplification du gène qui code pour l'AMPD que, dans la majorité des structures en échelle, les unités sont liées en orientation inverse et présentent une organisation très particulière dont la caractéristique la plus frappante est une distribution selon un ou plusieurs plans de symétrie successifs [23] (figure 2A). Cette organisation a ensuite été confirmée dans le cas de l'amplification du
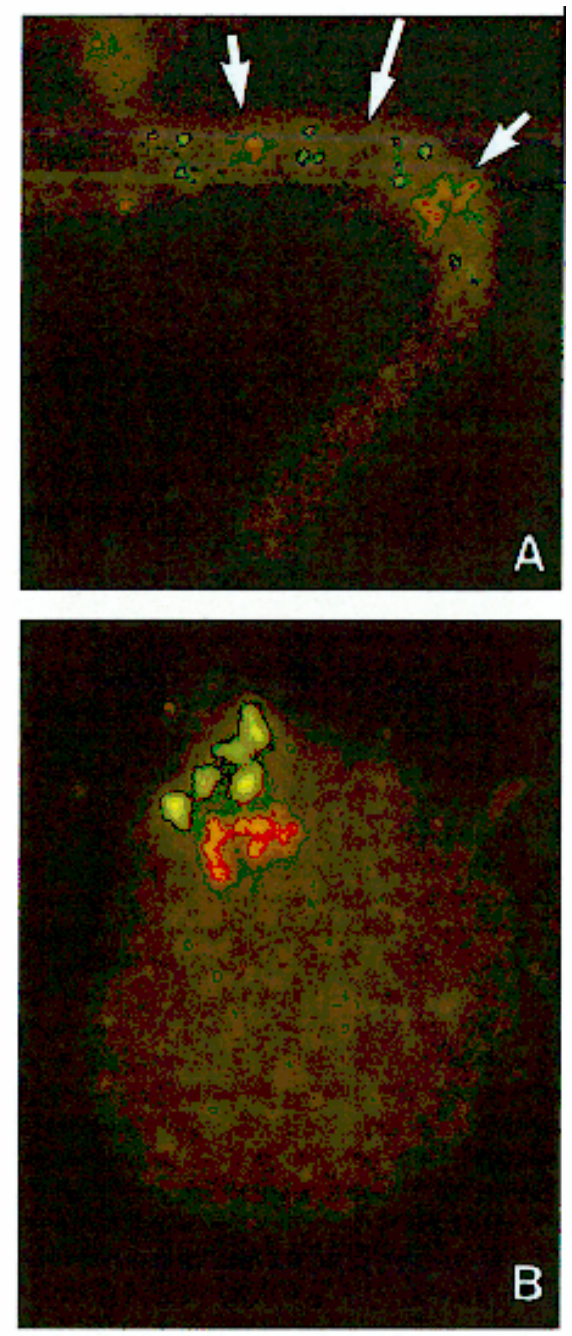

Figure 2. Images de FISH obtenues avec deux sondes révélant des séquences coamplifiées. A. Exemple de chromosome métaphasique montrant des unités amplifiées organisées en répétitions inverses et selon des plans de symétrie successifs (flèches). B. Exemple de noyau interphasique présentant une ségrégation des marqueurs dans des domaines différents.

gène qui code pour la DHFR [19]. Le seul mécanisme qui rende aisément compte des structures observées et de l'hétérogénéité clonale est l'opération de cycles de cassurefusion-pont de type chromatidien (CFP), initialement décrits par McClintock lorsqu'elle analysait les événements qui engendrent des délétions chez le maïs [25]. Si une cassure se produit dans un des chromo- 


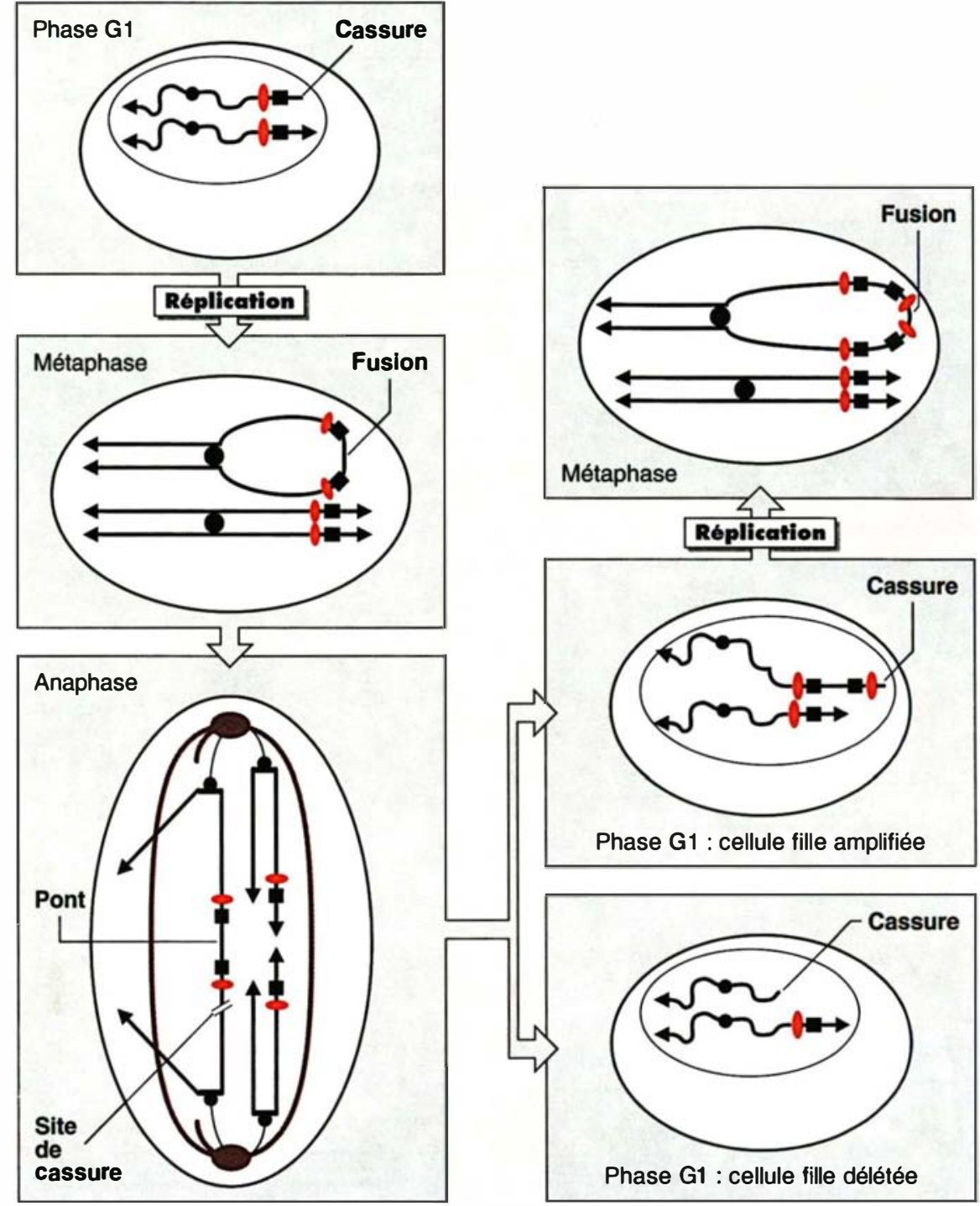

Figure 3. Mécanisme des cycles de cassure-fusion-pont (CFP) chromatidiens. Une seule paire de chromosomes homologues est représentée. Si une cassure survient dans le génome en phase G1, après réplication, les deux chromatides sœurs dépourvues de télomères fusionnent. En anaphase, un pont est formé quand les centromères fils migrent aux pôles opposés du fuseau mitotique; il s'ensuit une cassure qui donne naissance à une chromatide porteuse d'une duplication inverse et une chromatide délétée. Les cellules filles reçoivent chacune une chromatide normale et une chromatide cassée qui, à nouveau, sera à l'origine d'une fusion après réplication. Les ovales rouges et les carrés noirs figurent deux marqueurs coamplifiables, les flèches aux extrémités des chromatides schématisent les télomères, et les cercles noirs gros et petits - représentent les centromères des chromosomes ou des chromatides. somes d'une cellule en phase Gl, les deux chromatides sœurs formées après réplication sont dépourvues de télomères et fusionnent. Après clivage du centromère en mitose, un pont est formé par la chromatide dicentromérique résultant de la fusion. Une cassure survient nécessairement lorsque les deux centromères migrent en directions opposées le long du fuseau mitotique, le site de cette cassure étant supposé aléatoire. Chaque cellule fille reçoit un chromosome cassé qui va subir la même succession d'événements lors du cycle cellulaire suivant (figure 3). Le fait que le point de cassure soit spécifique pour chaque cellule et pour chaque cycle explique remarquablement bien la rapide hétérogénéisation des populations clonales, l'augmentation du nombre de copies dans certaines cellules au détriment de leurs cellules sœurs et l'hétérogénéité de taille des différentes unités d'un même tandem. L'étape de fusion des chromatides sours explique, quant à elle, la formation de palindromes et les symétries successives observées au sein du chromosome amplifié. Les intermédiaires prévus de ce mécanisme, chromosomes métaphasiques dont les chromatides sours sont fusionnées et ponts en ana-télophase, ont été observés sur les préparations cytologiques dans le cas du système AMPD [24]. Les événements susceptibles de stopper le processus ont été peu étudiés, cependant une analyse dans le système $\mathrm{CAD}$ suggère qu'un télomère peut, du moins dans certain cas, être resynthétisé de novo [26]. A priori, les réarrangements liés au fonctionnement des cycles de CFP chromatidiens n'affectent qu'un bras de chromosome. Cependant, dans les trois systèmes qui ont été étudiés aux stades précoces du phénomène, des réarrangements chromosomiques plus massifs, essentiellement des chromosomes métaphasiques dicentriques ou en anneau, ont été fréquemment détectés. Pour les systèmes $\mathrm{CAD}$ et $\mathrm{AMPD}$, deux types de chromosomes dicentriques ont été décrits : certains semblent résulter de la fusion de deux chromosomes qui portent la copie du gène sélectionné dans les cellules non amplifiées (dicentriques symétriques), d'autres de la fusion de ce même chromoso- 


\section{RÉFÉRENCES}

25. McClintock B. Chromosome organization and genic expression. Cold Spring Har bor Symp Quant Biol 1951 ; 16: 13-47.

26. Bertoni L, Attolini C, Tessera L, Mucciolo $\mathrm{E}$, Giulotto $\mathrm{E}$. Role of telomeric and non-telomeric (TTAGGG)n sequences in gene amplification and chromosome stability. Genomics 1994; $24: 53-62$.

27. Stark GR. Regulation and mechanisms of mammalian gene amplification. Adv Can cer Res $1993 ; 61: 87-113$.

28. McClintock B. Mutable loci in maize Camegie Inst Wash Year Book 1948; 47: 15569

29. Moore RC, Barber L, Bingham CG. Is it misrepair or lack of repair which kills cells irradiated in $G_{\text {? }}$ ? In : Obe G, Natarajan AT eds. Chromosomal aberrations, basic and applied aspects. Berlin : Springer Verlag, 1990 ; 41-9.

30. Martins MB, Sabatier L, Ricoul M, Pinton A, Dutrillaux B. Specific chromosome instability induced by heavy ions: a step towards transformation of human fibroblasts? Mutat Res 1993; 285: 229-37.

31. TienKuo M, Vyas RC, Jiang LX, Hittelman WN. Chromosome breakage at a major fragile site associated with P-glycoprotein gene amplification in multidrug-resistan CHO cells. Mol Cell Biol 1994; 14: 5202-11.

32. Miele M, Bonatti S, Menichini P, Ottaggio $\mathrm{L}$, Abbondandolo $\mathrm{A}$. The presence of amplified regions affects the stability of chromosomes in drug resistant Chinese hamster cells. Mutat Res 1989; 219; 171-8.

33. Nacheva E, Kearney L, Bower M, Chaplin T, Douek E, Das S, Young BD. In situ hybridization analysis of a homogeneously staining region at $11 \mathrm{q} 23-24$ in an acute myeloid leukaemia (M5) using yeast artificial chromosomes. Genes Chrom Cancer 1993; 7 : 123-7.

34. Roelofs H, Schuuring E, Wiegant J, Michalides R, Giphart-Gassler M. Amplification of the 1 lq13 region in human carcinoma cell lines: a mechanistic view. Genes Chrom Cancer 1993; $7: 74-84$.

35. Pedeutour F, Suijkerbuijk RF, Forus A VanGaal J, VandeKlundert W, Coindre JM, Nicolo G, Collin F, VnHaelst U, Huffermann K, Turc-Carel C. Complex composition and co-amplification of SAS and MDM2 in ring and giant rod marker chromosomes in well-differentiated liposarcoma.
Cellule engagée dans des cycles de CFP chromatidiens


Endoreduplication

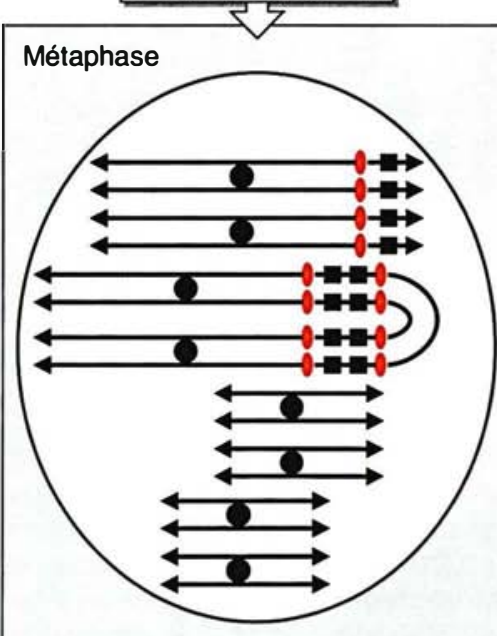

Chromosome dicentrique "symétrique" dans une cellule tétraploïde
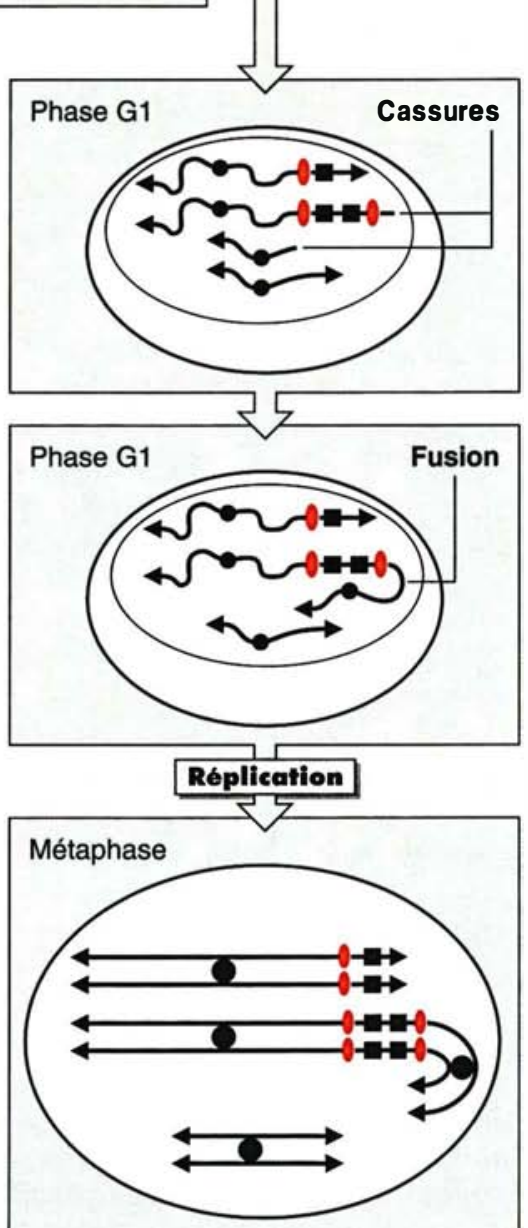

Chromosome dicentrique banal dans une cellule diploïde

Figure 4. Formation de chromosomes amplifiés dicentriques. Deux paires de chromosomes homologues sont représentées. A partir de cellules engagées dans des cycles de CFP chromatidiens - des cellules tétraploïdes contenant des chromosomes dicentriques symétriques sont engendrées par endoreduplication (schémas de gauche) -, si une cassure dans une chromatide quelconque survient en phase G1, les deux chromatides cassées fusionnent avant réplication et donnent naissance, après réplication, à des cellules contenant un chromosome dicentrique banal (schémas de droite). Les symboles sont les mêmes que dans la figure 3 . 
me avec un chromosome quelconque (dicentriques banals) [21, 24]. De tels réarrangements ont été observés dans une fraction des cellules de populations clonales qui présentent, dans la grande majorité des cellules, des structures caractéristiques du fonctionnement des cycles de CFP chromatidiens. Ces chromosomes dicentriques paraissent donc formés par des événements secondaires survenant pendant l'expansion des populations. Dans le système AMPD, les dicentriques symétriques n'ont été observés que dans des cellules polyploïdes - généralement tétraploîdes - alors que la formation des dicentriques banals n'est pas corrélée à un changement de ploïdie. Une analyse détaillée des deux catégories de chromosomes dicentriques a conduit aux modèles présentés dans la figure 4 [24].

\section{Un rôle central pour les cassures}

Une question importante qui reste actuellement non résolue concerne la nature des événements capables d'enclencher le processus. Le pontage de deux chromatides sœurs peut découler, soit d'une recombinaison en U entre ces chromatides [19, 20], soit de l'apparition d'extrémités atélomériques capables de fusionner après réplication. Dans ce dernier cas, le point de départ pourrait être un raccourcissement excessif des télomères [27] ou une cassure chromatidienne [19, 23, 24]. Un certain nombre d'observations suggèrent que les cassures sont effectivement capables de déclencher le processus d'amplification. McClintock a montré, dans les cellules somatiques de maïs, que des cycles de CFP chromatidiens sont amorcés par des cassures résultant de la mobilisation d'un transposon [28]. Des arguments supplémentaires ont été apportés par l'analyse des conséquences de cassures induites dans le génome de cellules de mammifères: (1) des intermédiaires de cycles de CFP et une instabilité chromosomique qui se maintient pendant plusieurs générations cellulaires sont observés après irradiation aux rayons $\mathrm{X}$ (pour revue, voir [29]), (2) les particules émises par le néon, l'argon, le plomb ou le xénon induisent des réarrangements chromosomiques non clonaux pendant une dizaine de générations cellulaires, puis ultérieurement des réarrangements clonaux, notamment des HSR [30], (3) lorsque des cassures sont induites préférentiellement au niveau d'un site fragile, proche du gène impliqué dans le phénomène de résistance croisée à de multiples médicaments (MDR, mutidrug resis tance) et en position télomérique par rapport à ce gène, une augmentation significative de la fréquence de mutants amplifiés pour ce locus est observée [31]. L'ensemble de ces résultats laisse penser que la cassure aléatoire d'une chromatide suffit à faire démarrer le processus d'amplification dans les cellules permissives.

Ce mécanisme de cycles de CFP chromatidiens rend compte de l'ensemble des observations effectuées dans des populations cellulaires engagées depuis peu dans un processus d'amplification intrachromosomique. En revanche, il n'explique pas à lui seul l'évolution rapide des structures en échelle vers des formes beaucoup plus compactes qui est observée au cours de l'expansion clonale. L'organisation topologique du noyau pourrait jouer un role déterminant dans le raccourcissement des unités répétées. En effet dans le système AMPD, l'observation des noyaux interphasiques aux stades précoces de l'amplification a confirmé des travaux antérieurs décrivant des déformations nucléaires fréquentes (bourgeons) dans des lignées de cellules amplifiées [32]. Ces bourgeons sont généralement marqués par les sondes qui détectent les séquences amplifiées, ce qui suggère que l'ADN excédentaire est difficilement empaqueté dans le noyau. En outre, ces bourgeons peuvent être extrudés et donner naissance à des micronoyaux $[23,32]$. Dans le système AMPD, le double marquage par des sondes révélant des séquences coamplifiables a montré que ces marqueurs, distribués en alternance sur les chromosomes contenant des échelles, ségrègent dans des domaines nucléaires différents lors de l'interphase [23] (figure $2 B$ ). Ces observations s'interprètent facilement si les séquences d'ADN correspondant aux deux marqueurs sont ancrées au squelette nucléaire au niveau de domaines spécifiques où les séquences identiques prove- nant de chacune des unités amplifiées se trouvent regroupées (figure 5A). Fréquemment, les bourgeons sont marqués par plusieurs copies d'un seul des deux marqueurs; s'ils donnent naissance à des micronoyaux, des cassures multiples surviendront dans l'ADN amplifié. Un modèle qui tient compte de ces cassures interphasiques, aboutissant à des délétions simultanées au niveau de chaque unité, a été proposé pour expliquer la rapide condensation des séquences amplifiées lors de l'expansion clonale [23] (figure 5B). Des cassures mitotiques constitueraient donc une étape clé du mécanisme d'amplification intrachromosomique alors que des cassures interphasiques seraient responsables de l'évolution des structures.

\section{Cycles de CFP, cassures et cancer}

Il est évidemment primordial de déterminer si le mécanisme de cycles de CFP chromatidiens et les événements secondaires qui en découlent opèrent in vivo. En particulier, on peut se demander si des structures typiques du fonctionnement de ce mécanisme peuvent être trouvées dans des cellules dérivées de tumeurs. L'analyse par FISH de l'organisation des HSR identifiées dans les cellules d'un patient atteint de leucémie myéloïde aiguë (LMA) et dans celles de divers carcinomes a été réalisée récemment. Elle a révélé que les unités répétées peuvent être longues de plusieurs $\mathrm{Mb}$ et que les HSR sont souvent localisées sur un bras de chromosome qui porte une copie des séquences correspondantes dans les cellules normales [33, 34]. L'analyse par FISH des séquences amplifiées dans des cellules de LMA et d'un liposarcome a également mis en évidence une disposition des signaux d'hybridation attendue d'une organisation symétrique [33, 35]. Bien qu'une analyse des structures formées précocement demeure impossible dans le cas des tumeurs, l'organisation de certaines HSR ressemble donc remarquablement aux structures engendrées par l'opération des cycles de CFP chromatidiens. De plus, les réarrangements secondaires associés au fonctionnement de ce mécanisme in vitro don- 


\section{RÉFÉRENCES}

36. Nowell PC. The clonal evolution of tumor cell populations. Science $1976 ; 194$ 23-8.

37. Hsu TC, Pathak S, Cailleau R, Cowles SR. Nature of nuclear projections in an adenocarcinoma of the breast. Lancet 1974; ii : 413.

38. German J. Bloom's syndrome II. The prototype of genome disorders predisposing to chromosome instability and cancer In: German J, ed. Chromosomes and cancer. New York: John Wiley and Sons, 1974: 60117.

39. Lu X, Lane DP. Differential induction of transcriptionally active p53 following UV or ionizing radiation: defects in chromosome instability syndromes? Cell $1993 ; 75$ : 765-78.

40. Roelofs H, Tasseron-deJong JG, vanderWal-Aker J, Rodenburg RJT, vanHouten GBM, vandePutte $P$, Giphart-Gassler $M$. Gene amplification in a human osteosarcoma cell line results in the persistence of the original chromosome and the formation of translocation chromosomes. Mutat Res 1992; 276 : 241-59.

41. Stahl F, Wettergren Y, Levan G. Amplicon structure in multi-drug-resistant murine cells: a non-rearranged region of genomic DNA corresponding to large circular DNA. Mol Cell Biol 1992; 12: 1179-87.

42. Federspiel NA, Beverley SM, Schilling JW, Schimke RT. Novel DNA rearrangements are associated with dihydrofolate reductase gene amplification. I Biol Chem reductase gene amplit
$1984 ; 259: 9127-40$.

43. Carroll SM, DeRose ML, Gaudray $P$, Moore CM, Needham-Vandevanter DR, Von Hoff DD, Wahl GM. Double minute chromosomes can be produced from precursors derived from a chromosomal deletion. Mol Cell Biol 1988; 8: 1525-33.

44. Pauletti G, Lai E, Attardi G. Early appearance and long term persistence of the submicroscopic extrachromosomal elements (amplisomes) containing the amplified DHFR genes in human cell lines. Proc Nall

nent naissance à des anomalies cytologiques qui sont observées couramment dans les cellules tumorales. Par exemple, la présence de chromosomes dicentriques ou en anneau est une propriété commune à différents types de cancers (pour revue, voir [36]), et des bourgeons nucléaires et micronoyaux ont été décrits dans des cellules de liposarcomes et d'adénocarcinomes mammaires [35, 37]. Dans le cas des liposarcomes, il a été établi que les bourgeons nucléaires et les micronoyaux contiennent des séquences d'un chromosome amplifié pour les oncogènes $S A S$ et $M D M 2$ [35]. D'autres arguments en faveur du fonctionnement des cycles de CFP chromatidiens au cours de l'oncogenèse viennent de l'étude cytogénétique de cellules d'individus atteints de maladies génétiques conduisant à une prédisposition au cancer. L'analyse réalisée sur le syndrome de Bloom est peut-être la plus suggestive puisque les intermédiaires métaphasiques et anaphasiques des cycles de CFP et des bourgeons nucléaires ont été observés dans les cellules prétumorales et tumorales des patients, conjointement aux aberrations chromosomiques spécifiques de ce syndrome [38]. Il a été également montré que la régulation du cycle cellulaire, en réponse aux cassures induites par les agents clastogènes, est perturbée dans les cellules de certains patients atteints de ce syndrome. Les cellules de malades atteints d'ataxie-télangectasie, un autre syndrome de prédisposition au cancer caractérisé par une fréquence élevée de chromosomes réarrangés, sont peut-être aussi déficientes dans ce type de régulation [39]. Le fait que les cassures chromosomiques jouent vraisemblablement un rôle primordial pour amorcer et propager l'amplification intrachromosomique permet d'imaginer comment un défaut d'activité de p53 (ou de toute autre protéine impliquée dans la même voie de régulation) peut déterminer la permissivité des cellules. En effet, dans une cellule normale, des lésions génomiques survenues en phase Gl induiront le processus d'apoptose ou seront réparées avant réplication, pendant l'arrêt de croissance au point de contrôle Gl/S. En revanche, des cellules dépourvues d'activité p53 entreront en phase S
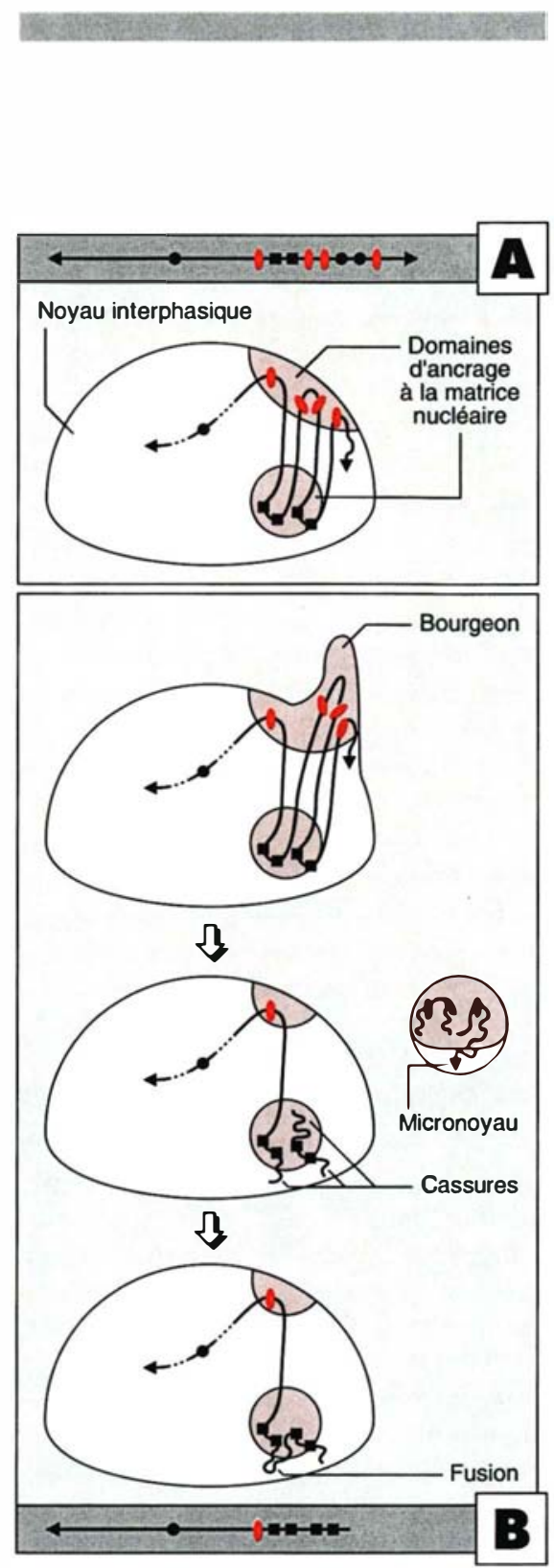

Figure 5. Organisation des noyaux interphasiques et modèle de raccourcissement des unités amplifiées. A. Comparaison de l'organisation d'une chromatide amplifiée en métaphase et en interphase. B. Cassures interphasiques résultant de l'extrusion d'un micronoyau qui englobe le domaine d'ancrage d'un des deux marqueurs seulement. Des délétions simultanées sont engendrées dans les unités d'un même tandem, et une chromatide dépourvue de télomère est reconstituée après fusion des extrémités libres, ce qui réamorce des cycles de CFP chromatidiens. Les symboles sont les mêmes que dans la figure 3. 

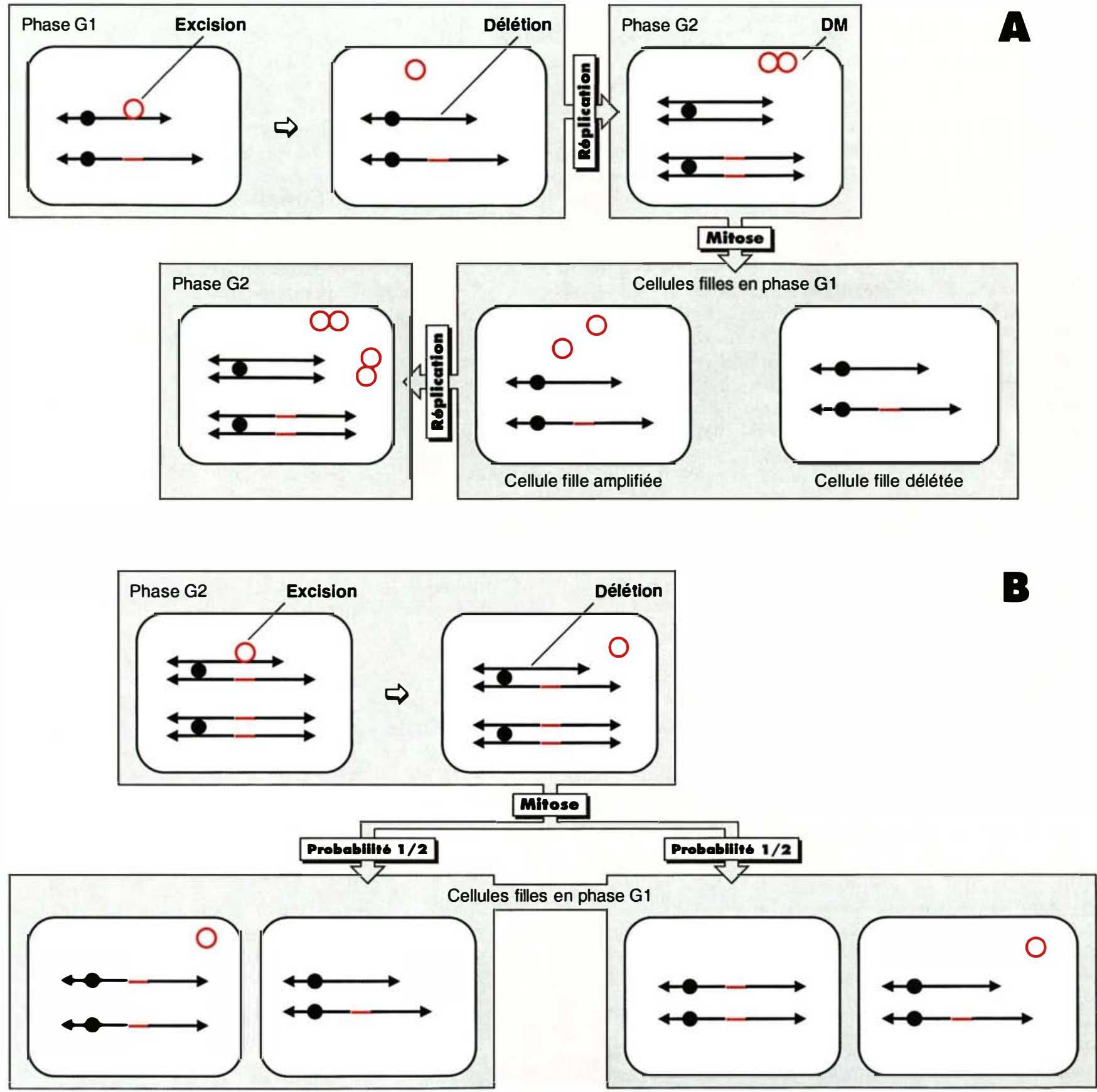

Figure 6. Modèle de formation des DM. A. Une molécule circulaire acentromérique est formée par excision dans une cellule en phase G1. Après réplication, un des homologues porte une délétion sur ses deux chromatides et un DM est engendré. L'absence de centromère conduit à une répartition au hasard des molécules extrachromosomiques à la mitose. Dans cet exemple, on a supposé qu'elles migrent toutes deux dans la même cellule fille après mitose; celle-ci possède donc trois copies de la séquence excisée tandis que sa cellule sœur n'en a plus qu'une. La ségrégation inégale des DM au cours des divisions successives conduira éventuellement à de hauts niveaux d'amplification dans certaines cellules de la population. B. Si une excision survient en phase G2, seule une chromatide sur les quatre porte une délétion. Après mitose, une des cellules filles héritera de cette délétion, l'autre sera normale au niveau chromosomique. La molécule extrachromosomique ayant une chance égale de ségréger dans l'une ou l'autre de ces cellules, une proportion égale de clones amplifiés composés de cellules portant une délétion de l'un des loci chromosomiques ou de cellules parfaitement diploïdes est attendue. Les symboles sont les mêmes que dans la figure 3. 
malgré leur génome endommagé, permettant la réplication d'une chromatide cassée, la fusion des chromatides sœurs atélomériques et le démarrage des cycles de CFP.

\section{Un mécanisme initial extrachromosomique}

Des DM ont été décrits aux stades précoces de l'amplification dans deux études seulement: le système DHFR, au cours de sélections effectuées à partir d'une lignée de hamster hémizygote pour ce lorus [17], et le système AMPD à partir d'une lignée diploïde pour la région concernée, mais dans ce cas les cellules présentant des DM apparaissent à une fréquence très faible comparée à celles qui portent des copies amplifiées intrachromosomiques [23, 24]. Les deux analyses ont montré que l'amplification peut s'accompagner d'une délétion d'une copie chromosomique du gène sélectionné; cette situation a été observée dans plus de la moitié des cas analysés pour les mutants portant une amplification extrachromosomique du gène $A M P D$. Dans ce même système, il a été établi que ces délétions au niveau d'un des homologues n'entraînent aucun autre réarrangement du bras chromosomique concerné. En particulier, la région qui s'étend de la fin de la délétion jusqu'au télomère est parfaitement conservée, contrairement à ce qui a été observé dans le cas de l'amplification par mise en œuvre de cycles de CFP chromatidiens. Ces résultats suggèrent que l'événement initial conduisant à une amplification extrachromosomique est l'excision d'une grande molécule circulaire acentrique, dont les copies pourraient ensuite être amplifiées au cours des divisions mitotiques par ségrégation inégale entre les cellules sœurs. Si cette molécule initiale est engendrée dans une cellule au stade $\mathrm{Gl}$, une délétion de l'une des copies chromosomiques sera nécessairement associée à sa formation (figure 6A). En revanche, si l'excision survient au stade $G 2$, la cellule fille qui recevra cet élément après mitose a une chance égale de porter ou non une délétion sur un de ses chromosomes (figure 6B) $[24,40]$. Dans les études effectuées sur différentes nant des DM, la délétion d'une des copies chromosomiques du gène amplifié a été observée dans certains cas alors que deux homologues normaux ont été identifiés dans d'autres. Ces deux situations dont l'interprétation à donné lieu à de nombreuses controverses sont en fait compatibles avec l'hypothèse d'excisions survenant en G1 ou en G2. Si les DM résultent effectivement de l'opération de ce mécanisme, leur séquence devrait être identique à celle du locus chromosomique initial, sauf au niveau de la jonction résultant de l'événement de recombinaison responsable de l'excision. Des résultats de cartographie à l'appui de cette hypothèse ont effectivement été obtenus avec une lignée de cellules tumorales de souris amplifiées pour le gène $M D R$ [41]. Dans un autre cas, des cartes indiquant la présence de très nombreux réarrangements ont été établies [42] ; la complexité pourrait être le résultat d'événements secondaires survenus pendant l'expansion des populations cellulaires. En effet, une évolution des structures extrachromosomiques a été mise en évidence dans certains systèmes où des éléments initialement submicroscopiques (appelés épisomes) fusionnent pour former d'abord de petits DM, puis de gros



Figure 7. Types d'événements contribuant à l'hétérogénéisation des populations clonales permissives pour l'amplification. Schéma représentant les différents événements qui peuvent survenir pendant l'expansion de populations cellulaires et conduire à des réarrangements génomiques couplés à une amplification (ou délétion) de certaines séquences.
DM aisément observables au microscope [43]. Cette situation n'est pas générale [44] mais elle pourrait expliquer les cas où l'analyse par FISH a révélé que chaque élément extrachromosomique porte plusieurs copies du gène sélectionné.

\section{Conclusion}

Cette revue, principalement focalisée sur l'analyse par FISH des structures engendrées précocement au cours de l'amplification de quatre loci différents dans des cellules de hamster, présente les résultats obtenus et les modèles qui en dérivent, sans prétention à l'exhaustivité. Ces études montrent qu'au moins deux mécanismes ségrégatifs indépendants, l'un intrachromosomique et l'autre extrachromosomique, peuvent permettre d'accumuler des copies surnuméraires aux stades précoces du processus d'amplification. L'addition des conséquences cytologiques des deux mécanismes (figure 7) correspond remarquablement à une large fraction des aberrations génomiques observées dans les cellules tumorales. L'ensemble des résultats indique qu'une large part de l'instabilité génomique associée à l'amplification est corrélée au processus intrachromosomique. Il reste à déterminer si la réintégration de $\mathrm{DM}$ en position ectopique est elle-même source de cassures et de remaniements. Il faut en outre noter que, dans les deux types de mécanismes, des cellules porteuses de délétions sont engendrées parallèlement aux cellules amplifiées. Dans certains cas, les mêmes mécanismes pourraient être responsables de la perte des gènes suppresseurs de tumeur et de l'amplification des oncogènes, et accélérer de ce fait la manifestation d'un phénotype malin

Remerciements

Le travail personnel des auteurs a bénéficié de la collaboration du professeur G. Buttin et du soutien de l'université Pierre-et-Marie-Curie, de la Ligue nationale contre le cancer (Comité de Paris et Comité national), de l'ARC, de la Fondation pour la recherche médicale française et du ministère de la Recherche.

TIRÉS À PART 


\section{Summary}

Genic amplification, genome plasticity and oncogenesis

The nature of mechanism(s) of mammalian gene amplification has been hotly discussed for over ten years and many models have been proposed. Recently, fluorescent in situ hybridization (FISH) has provided a powerful tool to study the early stages of mammalian gene amplification. The results obtained in three different model systems established that early amplification relies on unequal segregation of gene copies at mitosis, rather than on local over-replication, and that the early amplified structures are extremely unstable. The latter property focuses attention on the relationship between the molecular processes leading to amplification and those generating major genomic rearrangements. Genomic instability and oncogene amplification are indeed two frequent properties of cancer cells, which adds considerable interest to further exploration of amplification in model systems of cultured cells where the dynamic aspects of genome remodeling can be more conveniently analyzed. The recent demonstration, in two models of cultured mammalian cells, that gene amplification is prevented by the expression of the $p 53$ gene - a major tumor suppressor gene that appears to exert a general control on genomic stability - is of particular interest because it links amplification to oncogenesis, and stresses the likely relevance of results obtained on model systems of cultured cells to the mechanisms responsible for genomic instability in cancer cells. At least two different mechanisms are responsible for the initial steps of the amplification process in mammalian cells. Early stages of chromosomal gene amplification are characterized by a striking heterogeneity from cell to cell within a mutant clone. The extracopies are generally located on a chromosome arm where one copy maps in unamplified cells, and are organized as megabase-long inverted repeats with one or several orders of symmetry. These properties are consistent with an amplification mechanism involving chromatid breakage followed by the fusion - after replication - of the telomereless sister chromatids. This phenomenon is cyclic because the bridge formed between the sister chromatids leads to further breaks at mitosis. However, metaphase amplified dicentric and ring chromosomes, which are frequently observed in all model systems of mammalian amplification, are not intermediates of this mechanism. It was shown that these rearranged chromosomes are created when secondary events occur during expansion of populations of cells that undergo the cycles of chromatid breakage. Extrachromosomal copies of the amplified genes are also observed at early stages of gene amplification. FISH analysis of the cells that contain double-minute (DM) chromosomes reveals that looping out of a circular molecule appears to generate at least a significant part of DMs and thereby to initiate - through unequal segregation at mitosis - an independant pathway of gene amplification, alternative to the operation of the intrachromosomal mechanism. 